\title{
The Right Answer is Not Always Correct: Evaluating Student Knowledge
}

\author{
Scott Miller \\ University of Victoria \\ smiller@ece.uvic.ca
}

\begin{abstract}
Students are often evaluated on their ability to find a known solution to a well-defined problem, which does not accurately reflect the types of problems encountered in post-academic engineering. With the resources available today, students may learn how to produce a final answer without adequately understanding course material or how to apply it to realworld problems. Presented is a methodology for using the design process itself as a criteria to assess higher levels of student knowledge in engineering design courses. These criteria also help develop a student's professional practise skills and consideration of human factors in engineering design. The application of this methodology can help generate a student's mark that more accurately reflects their abilities based on the learning objectives of a course.
\end{abstract}

\section{Introduction}

Students are often evaluated on their ability to find a known solution to a problem, generate working code to meet a specification, or complete an engineering design project. What these strategies do not necessarily take into account are the student's knowledge and process used in order to answer each problem. With the emergence of on-line tools like Google, engineering-centric forums, and other collaborative tools, it is becoming increasingly easy for students to find an acceptable correct answer to their work, without gaining the important understanding gained from the process itself: what they are actually doing, and why they are doing it. This is of paramount important in engineering design courses, where students often have the ability to define a project or target themselves and work towards a working prototype. A student managing to create a working project does not directly correlate to the course material being understood or to engineering experience being gained.

It is therefore proposed that engineering design courses should have a higher weighting placed on the design process itself than on the deliverables requested for an assignment or project. This can be accomplished in many ways, including oral examinations of a student's work, documentation requirements explaining their design choices and any design changes between project milestones, higher weighting for code commenting and documentation, and social and human factors to be made explicit during the design process. These principles, including a strong focus on human and social engineering considerations have been applied to a variety of classes and will be discussed in detail.

The contributions of the paper are as fol- 
lows:

1. Presents the limitations of pure answerkey marking assessments;

2. Discusses design process-centric methods of evaluation and their benefits; and,

3. Promotes professional practise skills as a marking criteria.

The paper is organized as follows. Section 2 discusses the limitations to evaluation methods based only on set answers or prescribed solutions. Section 3 presents the case for assessment schemes to be based on an entire design flow over the project deliverable itself. Section 4 concludes the paper.

\section{Limitations of Answer-driven Assessment Techniques}

Common to engineering assessment techniques is the use of answer-driven marking schemes; whether multiple choice, longanswer mathematics, conceptual short paragraph answers, etc., all of these assessment techniques try to demonstrate a student's knowledge based on a prescribed answer key on a question-by-question basis. Although these techniques are very important and relevant, there are also limitations inherent to any small-scale prescribed questions.

Firstly, many styles of traditional classroombased assessment, such as solving textbookstyle problems on a test or exam can lead students to learn to answer problems by memorizing when to apply certain formulae or theorems without understanding when or why they should be applying them. Furthermore, it has been shown that even students who excel at classroom learning tend to focus on lower forms of learning than those who apply theory to laboratory and practical learning [1]. To further exacerbate this, there are many on-line resources now available to help students learn how to memorize derivations, formulae, and techniques, but these students then find themselves unable to apply them on their own in a broader context.

Secondly, many of these techniques target very narrow-scope problems that may be important for building upon over a student's academic career, such as basic circuit analysis, etc., but later on these students fail to connect these problems to the larger world picture. For example, many of these problems have a single solution, yet in real-world engineering there are many possible solutions that could be explored. Therefore, many of the assessment techniques based on simple problems may not prepare students for higher-level critical thinking and problem analysis that is essential to the engineering profession.

Thirdly, as a practical profession it is becoming increasingly important to include open-ended large-scale projects in the engineering curriculum to introduce students to practical problem solving and professional practise issues [2]. Such projects do not lend themselves very well to rigidly prescribed questions, and require other means of assessment to validate if a student actually understands the primary concepts of a course, or if they were able to answer narrowly-focused questions on paper.

A major limitation to all of these scenarios is that a student may be able to learn how to solve a particular style of question well without understand how to apply that knowledge. This leads to students with high marks being unable to adequately apply their skills to larger, less well defined engineering problems.

As an alternative to these traditional means of student assessment, marks can instead be given over a term to the design process of a project itself as opposed to narrow snapshots of a student's design flow. In other words: mark the process itself and not just the deliverables. Consequently, a student's mark will reflect not 
just their ability to solve a particular type of problem, but also their ability to apply it to problems on a larger scale. This is discussed in the next section.

\section{The Case for Process-centric De- sign Project Marking}

As well-defined problem-and-answer questions are not adequate in evaluating a student's open-ended design work, other assessment techniques are needed. When assigning open-ended projects, another possible pitfall is marking the deliverables of the project solely; this can lead to inflated marks from groups that use extensive on-line resources or find a solution to the project that may be far from optimal. A solution to these problems is to mark the design process and flow itself instead of strictly marking the deliverables.

Although there have been many design flow models proposed [3, 4], common to these models are steps such as requirements gathering, test plan development, design, implementation, testing and product maintenance. It is these steps and the student's process of applying these steps as well as other problemsolving techniques that should be included in a student's grading. By tracking a students process and forcing them to intentionally write out their design decisions, their actual progress on their design can be ascertained.

As an example of how marking the process can work, let us look at the following scenario. We have a design project assigned where a student must create a piece of software to be used in a developing nation to help the user understand local health issues. The student submits a web-based final project that resembles a Wiki with loads of textual information and levelupon-level of hyperlinks. The project may be attractively styled and meet the requirements and overall goal of the project, but there are many pieces to their design missing, such as:
- Why did the student use a Wiki-like structure when much of their user-base has not navigated such an interface?

- Why is the interface heavily textual when much of the user-base may not be literate, or there may be diverse dialects over the proposed area of deployment?

- What stick with Western-centric styles of interface when more efficient interfaces may be available to users without predisposition towards a more common one?

All of these questions raise possible design issues in the requirement, design, and implementation stages. Did the student consider language and computer literacy when building a Wiki? Did the student consider that networks will not be available in remote areas when they built a web application? The project's design and implementation raise questions about the student's design process itself and may lead the instructor to believe the student chose to solve the problem in a way that is comfortable to their skill-set without taking the user's needs into account. This should result in an assessment of the student's reasons for choosing such a system, and if necessary reducing the student's marks based on a poor design process.

Such considerations as listed above show how evaluating the design process itself can also bring human engineering factors and ethical considerations into the grading scheme. If a student's solution has potential ethical implications, such as the potential for harm or property loss, and such considerations are not addressed, the student's mark should reflect this. If the student has not considered the human user and their requirements out of their project, then the student has not properly completed their design assignment and therefore should be made aware of this. This provides a mechanism for injecting professional practise con- 
siderations into their design work - all of their decisions for their design should be active and conscious decisions and not left to chance, or selected as a matter of ease.

In order to adequately assess the design process itself, project milestones should be cumulative with marks assigned to the following:

- Project time budgeting and logging. Any discrepancies should be discussed and explained.

- Design decisions should all be clearly explained. Why was certain hardware or software selected? Why was the interface designed as-is? Why was the project segmented into a particular set of modules?

- Changes to design decisions over the term should be clearly documented and justified. If the final project does not match the documentation provided there is a design error.

- Applicable use-case and system diagrams must be included and match the design decisions.

- Theory and additional assessment techniques should be compared to their practical application in the design process. If the two are different, the student may need additional assessment to gauge why their design or theory do not show an appropriate correlation to each other.

- Clearly commented code with obvious function names, clear circuit diagrams, clear mechanical drawings with proper scaling, etc.

All of these artifacts can be used to assess if a student understands how to apply the fundamentals of a course to a practical design, as well as improving some of the professional practise skills, such as communication, documentation, and considering human and ethical factors in engineering design. This will give a clearer overall impression of a student's level of knowledge and will help them prepare for their post-academic careers by providing insight and guidance beyond well-defined problems that are not common in the engineering profession.

In addition to reviewing a students work as a time-based design flow, such artifacts can be useful to determine a student's areas of weakness in applying the theoretical aspects of class than traditional problems can. For example, if a student does not seem able to answer questions based on their design, they may be using external sources such as peers, on-line resources, and work from previous terms to complete their project. If there is any doubt, a short oral examination of their work is generally sufficient to determine if they are applying design principles to their work, or are just creating a project for marks without proper understanding of their work. Therefore, the student's mark is able to more accurately reflect their knowledge and skill-set than by simply grading a final answer itself.

\section{Conclusions}

When assigning a mark to represent a student's knowledge in course material, assessment techniques based on small-scale welldefined answers may not capture enough of a student's understanding enough to accurately reflect it. This is problematic in design-based courses, as getting an acceptable final answer does not mean the student actually understands their design fully. Instead, the design process itself can become a large part of the marking criteria, where students must document and justify their designs to demonstrate the application of their skills to the problem. This encourages students to better consider design factors, such as human and ethical engineering considerations and allows for a mark to be a more accurate reflection of a student's abilities. 


\section{References}

[1] Chia-Ching Lin and Chin-Chung Tsai.

"The Relationships between Students'

Conceptions of Learning Engineering

and their Preferences".

In: Journal of Engineering Education ().

[2] Canadian

Engineering Accrediation Board.

Engineers Canada. May 2010. URL:

http://www . engineerscanada.

ca/e/pr_accreditation.cfm.

[3] Winston Royce.

"Managing the Development of Large

Software Systems".

In: Proceedings of IEEE WESCON 26.

1970, pp. 1-9.

[4] Barry Boehm.

"A Spiral Model of Software

Development and Enhancement".

In: Proceedings of ACM SIGSOFT

Software Engineering Notes. 1986,

pp. 14-24. 